typeset using JPSJ.sty $<$ ver.1.0b $>$

\title{
Self-Consistent Second Order Perturbation Theory for the Hubbard Model in Two Dimensions
}

\author{
Hidekazu NoJIRIt \\ Department of Physics, Nagoya University, Furo-cho, Chikusa-ku, Nagoya 464-8602
}

(Received )

\begin{abstract}
We apply self-consistent second order perturbation theory (SCSOPT) with respect to the onsite repulsive interaction $U$ to study the Hubbard model in two dimensions. We investigate single particle properties of the model over the entire doping range at zero temperature. It is shown that as doping decreases toward half-filling $\omega$-mass enhancement factor increases, while $k$-mass enhancement factor decreases. The increase in $\omega$-mass enhancement factor is larger than the decrease in $k$-mass enhancement factor, so that total-mass is larger than that in the non-interacting case. When particle number density per unit cell $n$ is given by $0.64<n<$ 1.0 interaction enhances anisotropy of the Fermi surface, whereas at lower densities $n<0.64$ interaction suppresses anisotropy of it. Due to the decrease in $k$-mass enhancement factor the density of states (DOS) at the Fermi level is suppressed. It is possible to understand the results within the framework of the weak coupling Fermi liquid theory.
\end{abstract}

KEYWORDS: Hubbard model, two dimensions, Fermi liquid, self consistent, second order perturbation, Fermi surface, $k$-mass, $\omega$-mass

\section{$\S 1$. Introduction}

Since the discovery of the high- $T_{\mathrm{c}}$ superconducting cuprates (HTSC), it two dimensional layered materials have been attracting the attention of many researchers. Two dimensional Hubbard model is one of the promising model to understand such materials. Investigations of the Hubbard model have a long history and the understanding of the one dimensional 3 ) and the infinite dimensional cases $3^{3}$ ) is in a higher level. In the case of one dimension DOS at the Fermi level becomes zero with infinitesimal interaction $U$. On the other hand in the case of infinite dimensions DOS at the Fermi level doesn't change at all until metal-insulator transition happens due to the locality of the self-energy. For the case of two dimensions the feature of the model hasn't been sufficiently elucidated.

We use here the self-consistent second order perturbation theory (SCSOPT) with respect to the on-site repulsive interaction $U$ to study the Hubbard model in two dimensions. The SCSOPT satisfies the Luttinger sum rule, which is necessary to construct the Fermi liquid theory 1 The SCSOPT was formerly used for studying the infinite dimensional case and was known to describe the low energy excitations of the paramagnetic metallic phase of the model correctly in the weak coupling case. our attention to the metallic phase of the model without magnetic order, which is suggested for a non-half- filling case in Quantum Monte Carlo simulations.6.8. On the contrary to infinite dimensional case the selfenergy of the two dimensional Hubbard model has $k$ dependence. The $k$-dependence of the self-energy is considered to play an important role in Mott transition in two dimensional systems such as $\mathrm{Ca}_{1-\mathrm{x}} \mathrm{Sr}_{\mathrm{x}} \mathrm{VO}_{3}$ (9) and HTSC 10) The $k$-dependence effects of the two dimensional Hubbard model were examined so far by second order perturbation theory (SOPT) 11 22 13) and the fluctuation exchange (FLEX) approximation.14.15) In the case of SOPT it was reported that for sufficiently large $U n(\mu)$ exceeds unity in spite of $\mu<0$ around $\mu \simeq 0$, which leads to unphysical negative charge susceptibility. ${ }^{13}$ Then we define $U_{1}(\mu)$ so that in the region $U>U_{1}$ SOPT is unphysical. In addition SOPT satisfies the Luttinger sum rule only approximately for small $U$ except for half-filling. This disagreement between the Fermi surface volume and the expectation value of the number operator is also remarkable near the half-filling. Then we define $U_{2}$ so that in the region $U>U_{2}$ physical features are affected by that disagreement. We can use SOPT only in the region $U<\min \left\{U_{1}, U_{2}\right\}$. There are not such limitations in SCSOPT. Meanwhile the FLEX approximation includes SCSOPT diagrams, but it would be meaningful to distinguish the contribution from SCSOPT diagrams from the contribution from the other diagrams.

We adopt the unit system in which $\hbar=1$ and $k_{\mathrm{B}}=1$.

* E-mail: j45747a@nucc.cc.nagoya-u.ac.jp 


\section{§2. Model and Calculations}

The Hubbard Hamiltonian is

$$
H=-t \sum_{n . n ., \sigma}\left(c_{i \sigma}^{\dagger} c_{j \sigma}+\text { h.c. }\right)+U \sum_{i} n_{i \downarrow} n_{i \uparrow},
$$

where $t$ is the transfer integral between the nearest neighbor sites and $U$ stands for the on-site repulsive interaction.

The one particle Green's function at zero temperature is defined as 16

$$
G_{\boldsymbol{R}_{\sigma}}(t) \equiv-\mathrm{i}<T \psi_{\sigma}(\boldsymbol{R}, t) \psi_{\sigma}^{\dagger}(\boldsymbol{O}, 0)>,
$$

where $\psi_{\sigma}(\boldsymbol{R}, t)$ denotes a particle field of electrons and $\langle\ldots\rangle$ and $T$ mean thermodynamic average over grand canonical ensemble and a time ordering operator, respectively.

The Green's function in $(\boldsymbol{k}, \omega)$ space can be expressed by the self-energy as

$$
G_{\boldsymbol{k}_{\sigma}}(\omega)=\frac{1}{\omega-\xi_{\boldsymbol{k}}-\Sigma_{\boldsymbol{k}_{\sigma}}(\omega)},
$$

where

$$
\xi_{\boldsymbol{k}}=\varepsilon_{\boldsymbol{k}}-\mu
$$

and

$$
\varepsilon_{\boldsymbol{k}}=-2 \cos \left(k_{x}\right)-2 \cos \left(k_{y}\right) .
$$

We define here the retarded self-energy and the retarded Green's function as

$$
\Sigma_{\boldsymbol{k}}^{(R)}(\omega) \equiv \begin{cases}\Sigma_{\boldsymbol{k}}(\omega) & \omega>0 \\ \Sigma_{\boldsymbol{k}}^{*}(\omega) & \omega<0\end{cases}
$$

and

$$
G_{\boldsymbol{k}_{\sigma}}^{(R)}(\omega) \equiv \frac{1}{\omega-\xi_{\boldsymbol{k}}-\Sigma_{\boldsymbol{k}_{\sigma}}^{(R)}(\omega)} .
$$

The Green's function can be expressed by the spectral function as

$$
G_{\boldsymbol{k}_{\sigma}}(\omega)=\int_{-\infty}^{\infty} \mathrm{d} E \frac{A_{\boldsymbol{k}_{\sigma}}(E)}{\omega-E-\mathrm{i} \delta \operatorname{sign}(E)},
$$

where

$$
A_{\boldsymbol{k}_{\sigma}}(E) \equiv-\frac{1}{\pi} \operatorname{Im} G_{\boldsymbol{k}_{\sigma}}^{(R)}(E) .
$$

Then it follows

$$
\begin{aligned}
G_{\boldsymbol{k}_{\sigma}}(t) & =\mathrm{i} \theta(-t) \int_{-\infty}^{0} \mathrm{~d} E e^{-\mathrm{i} E t+\delta t} A_{\boldsymbol{k}_{\sigma}}(E) \\
& -\mathrm{i} \theta(t) \int_{0}^{\infty} \mathrm{d} E e^{-\mathrm{i} E t-\delta t} A_{\boldsymbol{k}_{\sigma}}(E) \\
& =\mathrm{i} \theta(-t) a_{\boldsymbol{k}_{\sigma}}(t)-\mathrm{i} \theta(t) b_{\boldsymbol{k}_{\sigma}}(t)
\end{aligned}
$$

Here we define

$$
a_{\boldsymbol{k}_{\sigma}}(t) \equiv \int_{-\infty}^{0} \mathrm{~d} E e^{-\mathrm{i} E t+\delta t} A_{\boldsymbol{k}_{\sigma}}(E)
$$

and

$$
{ }^{b} \boldsymbol{k}_{\sigma}(t) \equiv \int_{0}^{\infty} \mathrm{d} E e^{-\mathrm{i} E t-\delta t} A_{\boldsymbol{k}_{\sigma}}(E) .
$$

Real space Green's functions can be calculated as

$$
G_{\boldsymbol{R} \sigma}(t)=\frac{1}{N_{L}} \sum_{\boldsymbol{k}} G_{\boldsymbol{k}_{\sigma}}(t) e^{\mathrm{i} \boldsymbol{k} \cdot \boldsymbol{R}} .
$$

The contribution of the Hartree diagram (Fig. 1) to the self-energy is

$$
\Sigma_{\boldsymbol{k}_{\sigma}}^{(\text {Hartree) }}(\omega)=U n_{-\sigma} .
$$

As we consider the paramagnetic state in the present paper,

$$
\Sigma_{\boldsymbol{k}_{\sigma}}^{(\mathrm{Hartree})}(\omega)=U \frac{n}{2} .
$$

This static contribution to the self-energy can be incorporated into the chemical potential, and as a result the redefined chemical potential takes the value 0 at halffilling.

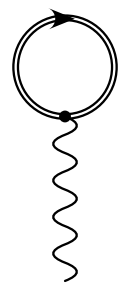

Fig. 1. Hartree diagram. The wavy and double solid lines represent the on-site repulsive interaction and the exact one particle Green's function, respectively.

The second order contribution (Fig. 2) to the selfenergy is

$$
\Sigma_{\boldsymbol{R}_{\sigma}}^{(2)}(t)=U^{2} G_{\boldsymbol{R}_{\sigma}}^{(0)}(t) G_{\boldsymbol{R}_{-\sigma}}^{(0)}(t) G_{-\boldsymbol{R}_{-\sigma}}^{(0)}(-t),
$$

where $G_{\boldsymbol{R}_{\sigma}}^{(0)}(t)$ is the Green's function of the noninteracting system. Similarly the SCSOPT contribution (Fig. 3) to the self-energy is

$$
\Sigma_{\boldsymbol{R}_{\sigma}}^{(s c)}(t)=U^{2} G_{\boldsymbol{R}_{\sigma}}^{(s c)}(t) G_{\boldsymbol{R}_{-\sigma}}^{(s c)}(t) G_{-\boldsymbol{R}_{-\sigma}}^{(s c)}(-t),
$$

where

$$
G_{\boldsymbol{k}_{\sigma}}^{(s c)}(\omega)=\frac{1}{\omega-\xi_{\boldsymbol{k}}-\Sigma_{\boldsymbol{k}_{\sigma}}^{(s c)}(\omega)} .
$$

In real space the retarded self-energy of SCSOPT is expressed as

$$
\begin{aligned}
\Sigma_{\boldsymbol{R}_{\sigma}(s)}^{(R)}(t) & =-\mathrm{i} U^{2} \theta(t)\left\{b_{\boldsymbol{R}_{\sigma}}(t) b_{\boldsymbol{R}_{-\sigma}}(t) a_{-} \boldsymbol{R}_{-\sigma}(-t)\right. \\
& \left.+{ }^{a} \boldsymbol{R}_{\sigma}(t) a_{\boldsymbol{R}_{-\sigma}}(t) b_{-} \boldsymbol{R}_{-\sigma}(-t)\right\} \\
& =U^{2} \theta(t)\left\{G_{\boldsymbol{R}_{\sigma}}^{(s c)}(t) G_{\boldsymbol{R}_{-\sigma}}^{(s c)}(t) G_{-\boldsymbol{R}_{-\sigma}}^{(s c)}(-t)\right.
\end{aligned}
$$




$$
\left.+G_{-\boldsymbol{R}_{\sigma}}^{(s c) *}(-t) G_{-\boldsymbol{R}_{-\sigma}}^{(s c) *}(-t) G_{\boldsymbol{R}_{-\sigma}}^{(s c)}(t)\right\}
$$

The real and the imaginary parts of the retarded selfenergy satisfy the Kramers-Kronig (K.K.) relation,

$$
\operatorname{Re} \Sigma_{\boldsymbol{k}_{\sigma}}^{(R)}(\omega)=\frac{1}{\pi} P \int_{-\infty}^{\infty} \mathrm{d} \omega^{\prime} \frac{\operatorname{Im} \Sigma_{\boldsymbol{k}_{\sigma}}^{(R)}\left(\omega^{\prime}\right)}{\omega^{\prime}-\omega}
$$

The algorithm for numerical calculations is as follows.

(1) We choose the initial $\Sigma_{\boldsymbol{k}_{\sigma}}^{(R)}(\omega)$, for example, the selfenergy of SOPT.

(2) We calculate $G_{\boldsymbol{R}_{\sigma}}(t)$ using eqs. (2.5), (2.7), (2.8) and (2.11). At this time, since $A_{\boldsymbol{k}}(\omega)$ is localized with respect to energy within the extent of the band width, the integration in eq. (2.8) can be reduced to an appropriate finite energy interval.

(3) Using eq. (2.17) we calculate $\Sigma_{\boldsymbol{R}_{\sigma}}^{(R)}(t)$ which goes to zero more rapidly than $\Sigma_{\boldsymbol{R}_{\sigma}}(t)$ as $t$ increases.

(4) We calculate $\Sigma_{\boldsymbol{k}_{\sigma}}^{(R)}(\omega)$ using the Fast Fourier Transformation (FFT). At this time it is efficient to calculate first $\operatorname{Im} \Sigma_{\boldsymbol{k}_{\sigma}}^{(R)}(\omega)$ which is localized and calculate $\operatorname{Re} \Sigma_{\boldsymbol{k}_{\sigma}}^{(R)}(\omega)$ using the K.K. relation eq. 2.18).

(5) If we iterate this procedure until convergence occurs, we obtain the SCSOPT $\Sigma_{\boldsymbol{k}_{\sigma}}^{(R)}(\omega)$.

Here we present the results for a lattice with $256 \times 256$ sites and the anti-periodic boundary condition. The FFT from time to energy variable was performed on 512 points. The energy spacing $\delta \omega$ is 0.06 and the momentum spacing $\delta k \equiv 2 \pi / 256$ is 0.025 . All of the numerical results are calculated for zero temperature. A typical run to solve the Hubbard model with a fixed chemical potential value takes 1 hour on a DEC alpha station 250 $4 / 266$.

Hereafter we drop the spin indices for physical quantities as we are in the paramagnetic phase.

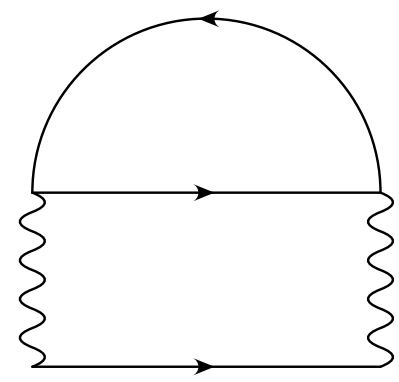

Fig. 2. Diagrammatic representation of the self-energy in second order in $U$. The wavy and the solid lines represent on-site repulsive interactions and free Green's functions, respectively.

\section{$\S 3 . \quad$ Results}

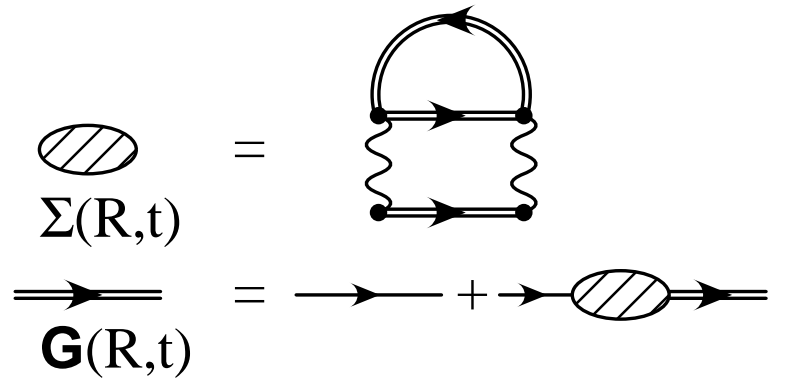

Fig. 3. Diagrammatic representation of SCSOPT. The solid, the double solid, and the wavy lines represent free Green's functions, renormalized Green's functions, and on-site repulsive interactions, respectively.

\subsection{Chemical potential shift}

As a function of density the interaction-induced chemical potential shifts are shown in Fig. 4 . The chemical potential doesn't shift for half-filling $(n=1)$ case due to particle-hole symmetry, and has a maximal shift -0.261 at $n=0.47$ for $U=4$.

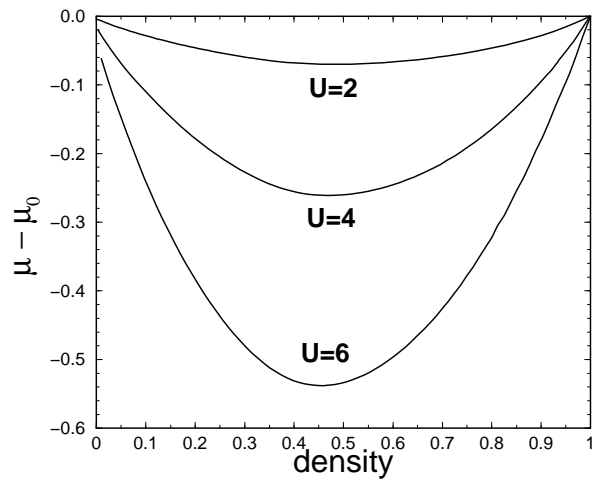

Fig. 4. Chemical potential shifts v.s. particle density. $\mu$ and $\mu_{0}$ represent the chemical potential of the interacting and the noninteracting system, respectively.

In the weak coupling theory the chemical potential shift is related with the $\omega=0$ self-energy on the noninteracting Fermi surface as 17

$$
\delta \mu \simeq<\operatorname{Re} \Sigma_{\boldsymbol{k}_{F 0}}(0)>_{F S 0},
$$

where $\boldsymbol{k}_{F 0}$ is the Fermi momentum of the non-interacting system and $\langle\ldots\rangle_{F S 0}$ means the average over the noninteracting Fermi surface

$$
<\operatorname{Re} \Sigma_{\boldsymbol{k}_{F 0}}(0)>_{F S 0} \equiv \frac{\sum_{\boldsymbol{k}} \delta\left(\epsilon_{\boldsymbol{k}}-\mu_{0}\right) \operatorname{Re} \Sigma_{\boldsymbol{k}_{F 0}}(0)}{\sum_{\boldsymbol{k}} \delta\left(\epsilon_{\boldsymbol{k}}-\mu_{0}\right)}
$$

where $\mu_{0}$ is the unperturbed chemical potential.

Figure 5 shows the $\operatorname{SCSOPT} \operatorname{Re} \Sigma_{\boldsymbol{k}_{F 0}}(0)$ as a func- 
tion of $n$. The curve of chemical potential shift is put between the curve of $\operatorname{Re} \Sigma_{\boldsymbol{k}_{F 0}}(0)$ of $(\pi, 0)$ direction and that of $(\pi, \pi)$ direction. The variation of $\operatorname{Re} \Sigma_{\boldsymbol{k}_{F 0}}(0)$ on the non-interacting Fermi surface is much smaller than $\operatorname{Re} \Sigma_{\boldsymbol{k}_{F 0}}(0)$ itself. Therefore we can conclude that the deviation of $\delta \mu$ from eq. (3.1) is small compared with $\delta \mu$ itself.

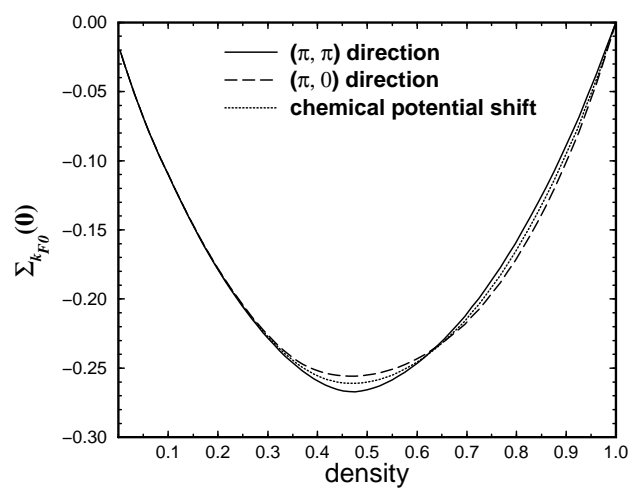

Fig. 5. $\Sigma_{\boldsymbol{k}_{F 0}}(0)$ of SCSOPT as a function of $n$ for interaction strength $U=4$. The results are shown for $(\pi, 0)$ and $(\pi, \pi)$ directions on the non-interacting Fermi surface. Chemical potential shift for the same interaction strength is also shown for comparison.

\subsection{Fermi surface}

The excitation energy of the quasi-particle is determined by the secular equation

$$
E_{\boldsymbol{k}}-\xi_{\boldsymbol{k}}-\operatorname{Re} \Sigma_{\boldsymbol{k}}\left(E_{\boldsymbol{k}}\right)=0
$$

The Fermi surface of the interacting system is determined by the condition $E_{\boldsymbol{k}}=0$, hence

$$
\xi_{\boldsymbol{k}_{F}}+\operatorname{Re} \Sigma_{\boldsymbol{k}_{F}}(0)=0,
$$

where $\boldsymbol{k}_{F}$ is the Fermi momentum of the interacting system.

As shown in Fig. 6 at densities $0.64<n<1.0$ the Fermi momentum of the interacting system is larger than that of the non-interacting system in $(\pi, 0)$ direction, whereas in $(\pi, \pi)$ direction it is smaller than that of the non-interacting system. On the other hand at densities $n<0.64$ the Fermi momentum of the interacting system is smaller than that of the non-interacting system in $(\pi, 0)$ direction, whereas in $(\pi, \pi)$ direction it is larger than that of the non-interacting system.

Figure 7 shows the Fermi surface deformation for various densities. $\delta k_{F}$ is defined as

$$
\delta k_{F}\left(\boldsymbol{k}_{F 0}\right) \equiv\left(\boldsymbol{k}_{F}-\boldsymbol{k}_{F 0}\right) \cdot \boldsymbol{n}_{\boldsymbol{k}_{F 0}},
$$

where we take the non-interacting Fermi momentum $\boldsymbol{k}_{F 0}$ so that $\boldsymbol{k}_{F}-\boldsymbol{k}_{F 0}$ is perpendicular to the non-interacting
Fermi surface, and $\boldsymbol{n}_{\boldsymbol{k}_{F 0}}$ is a unit vector normal to the non-interacting Fermi surface and always is directed outwards from $\boldsymbol{k}_{F 0}$. $\phi$ represents the polar angles of positions on the non-interacting Fermi surface in units of degree, which is described as $\operatorname{Arctan}\left(k_{F 0 y} / k_{F 0 x}\right)$ mathematically. At densities 0.9, 0.8 and 0.7 , which are larger than $0.64 \delta k_{F}$ is monotonously decrease as $\phi$ changes from $0^{\circ}$ to $45^{\circ}$. On the other hand at densities 0.5 and 0.3 , which are smaller than $0.64 \delta k_{F}$ is monotonously increase in that region of $\phi$. Then there is a unique intersection of interacting and non-interacting Fermi surfaces in that region of $\phi$ except for the cases of $n=1$ (due to particle-hole symmetry) and 0.64. That intersection moves from $0^{\circ}$ to $22.5^{\circ}$ monotonously as $n$ changes from 1 to 0 .

Then from Figs. 6 and 7 it is concluded that at densities $0.64<n<1.0$ interaction enhances anisotropy of the Fermi surface, whereas at densities $n<0.64$ it suppresses anisotropy.

Fermi surface deformation is very small and the topology doesn't change.

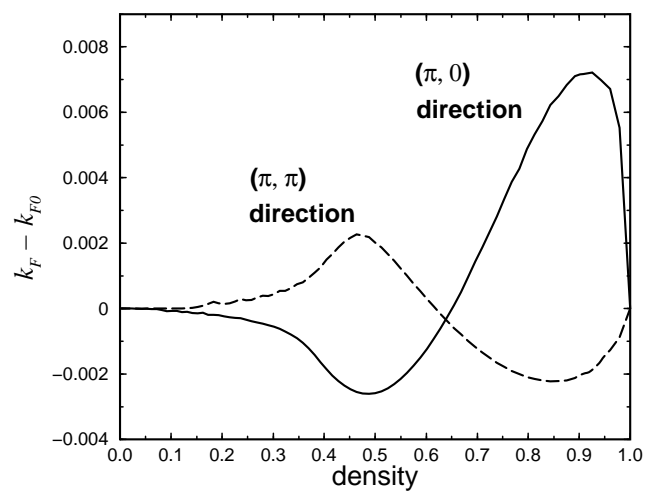

Fig. 6. Fermi momentum shifts induced by the interaction over the entire doping range in SCSOPT. $k_{F}$ and $k_{F 0}$ denote the Fermi momentum of the interacting and the non-interacting system, respectively. The results are shown for $(\pi, 0)$ and $(\pi, \pi)$ directions on the Fermi surface. Interaction strength $U=4$.

In the weak coupling case eq. (3.4) is reduced to 17

$$
\delta k_{F}\left(\boldsymbol{k}_{F 0}\right) \simeq \frac{\delta \mu-\operatorname{Re} \Sigma_{\boldsymbol{k}_{F 0}}(0)}{v_{\boldsymbol{k}_{F 0}}^{0}}
$$

where $v_{\boldsymbol{k}_{F 0}}^{0} \equiv\left|\nabla \varepsilon \boldsymbol{k}_{F 0}\right|$ is the Fermi velocity of the noninteracting system. Putting eq. (3.1) into eq. (3.6) we get

$$
\delta k_{F}\left(\boldsymbol{k}_{F 0}\right) \simeq \frac{<\operatorname{Re} \Sigma_{\boldsymbol{k}_{F 0}}(0)>_{F S 0}-\operatorname{Re} \Sigma_{\boldsymbol{k}_{F 0}}(0)}{v_{\boldsymbol{k}_{F 0}}^{0}}
$$

Halboth and Metzner calculated the second order coefficient of $\delta k_{F}$ in $U$ using eq. (3.7) in SOPT.17) The 


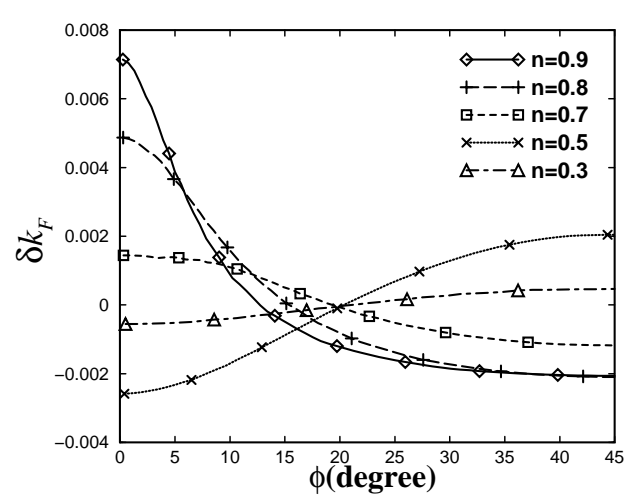

Fig. 7. Fermi surface deformation induced by the interaction for various densities. The horizontal axis represents the polar angles of positions on the non-interacting Fermi surface in units of degree. Interaction strength $\mathrm{U}=4$.

results of Fig. 7 are almost the same as the results of Halboth and Metzner except for the scale of the $y$-axis. This means that in SCSOPT eqs. (3.1) and (3.6) are in fact satisfied approximately and low energy part $<\Sigma_{\boldsymbol{k}_{F 0}}^{(2)}\left(0, \mu_{0}(n)\right)>_{F S 0}-\Sigma_{\boldsymbol{k}_{F 0}}^{(2)}\left(0, \mu_{0}(n)\right)$ used in their work is almost the same as $<\Sigma_{\boldsymbol{k}_{F 0}}^{(s c)}(0, \mu(n))>_{F S 0}$ $-\Sigma_{\boldsymbol{k}_{F 0}}^{(s c)}(0, \mu(n))$ except for the scale. The difference in the scale originates in the fact that in SOPT $\delta k_{F}$ increases in proportion to $U^{2}$, whereas in SCSOPT it isn't in proportion to $U^{2}$.

As shown in Fig. 5 at densities $0.64<n<1.0$ $\operatorname{Re} \Sigma_{\boldsymbol{k}_{F 0}}(0)$ in $(\pi, 0)$ direction is smaller than that of $(\pi, \pi)$ direction, whereas at low densities $n<0.64$ it is larger than that of $(\pi, \pi)$ direction. These features combined with eq. (3.7) account for the results in Fig. 6.

\section{$3.3 k$-mass and $\omega$-mass}

The denominator of the Green's function is expanded around $E_{\boldsymbol{k}}$ as

$\omega-\xi_{\boldsymbol{k}}-\operatorname{Re} \Sigma_{\boldsymbol{k}}(\omega) \simeq\left(1-\left.\frac{\partial \operatorname{Re} \Sigma_{\boldsymbol{k}}(\omega)}{\partial \omega}\right|_{\omega=E_{\boldsymbol{k}}}\right)\left(\omega-E_{\boldsymbol{k}}\right)$.

Here we define $\omega$-mass and $\omega$-mass enhancement factors as

$$
\gamma_{\omega} \equiv \frac{m_{\omega}}{m_{o}} \equiv 1-\left.\frac{\partial \operatorname{Re} \Sigma_{\boldsymbol{k}_{F}}(\omega)}{\partial \omega}\right|_{\omega=0} \equiv \frac{1}{Z_{\boldsymbol{k}_{F}}},
$$

where $m_{o}$ is the mass in the case of $U=0$

$$
\frac{k_{F}}{m_{o}} \equiv\left|\nabla \xi_{\boldsymbol{k}_{F}}\right| \text {. }
$$

We define $k$-mass and $k$-mass enhancement factors as

$$
\frac{k_{F}}{m_{k}} \equiv \mid \operatorname{grad}_{\boldsymbol{k}}\left(\xi_{\boldsymbol{k}}+\operatorname{Re} \Sigma_{\boldsymbol{k}}(0)\right) \|_{\boldsymbol{k}=\boldsymbol{k}_{F}}
$$

and

$$
\gamma_{k} \equiv \frac{m_{k}}{m_{o}} .
$$

Dispersion of the quasi-particle energy is expressed in terms of mass enhancement factors as

$$
E_{\boldsymbol{k}}=\frac{k_{F}}{m_{0} \gamma_{k} \gamma_{\omega}}\left(\boldsymbol{k}-\boldsymbol{k}_{F}\right) \cdot \boldsymbol{n}_{\boldsymbol{k}_{F}},
$$

where we take $\boldsymbol{k}_{F}$ so that $\boldsymbol{k}-\boldsymbol{k}_{F}$ is perpendicular to the Fermi surface and $\boldsymbol{n}_{\boldsymbol{k}_{F}}$ is a unit vector normal to the Fermi surface and always is directed outwards from $\boldsymbol{k}_{F}$.

Figure 8 shows that as $n$ increases toward half-filling $\omega$-mass enhancement factor increases, while $k$-mass enhancement factor decreases (Exceptionally $\gamma_{\omega}\left(90^{\circ}\right)$ increases slightly in the region $0.81<n<0.87)$. The amounts of the increase in $\omega$-mass enhancement factor and the decrease in $k$-mass enhancement factor are larger in $(0, \pi)$ direction than those in $(\pi, \pi)$ direction.

From eq. (3.13) total mass enhancement factor is determined by $\gamma_{\omega} \gamma_{k}$. The increase in $\omega$-mass enhancement factor is larger than the decrease in $k$-mass enhancement factor, so that total mass is larger than that of the non-interacting system. As shown in Fig. 9 total mass enhancement factor in $(0, \pi)$ direction has a peak at $n=0.97$, and decreases for $n>0.97$ due to the decrease in $k$-mass enhancement factor.

The decrease in $k$-mass in this weak coupling theory is not sufficient to explain the experimental data of Mott-Hubbard systems such as $\mathrm{Ca}_{1-\mathrm{x}} \mathrm{Sr}_{\mathrm{x}} \mathrm{VO}_{3}$, , HTSC $\left.\mathrm{La}_{2-\mathrm{x}} \mathrm{Sr}_{\mathrm{x}} \mathrm{CuO}_{4}{ }^{10}\right)$ and an organic conductor BEDT-TTF salt $\alpha-(\mathrm{ET})_{2} \mathrm{KHg}(\mathrm{SCN})_{4}$. 18

Recently Yoda and Yamada 19 investigated the effect of the long-range Coulomb interaction on the $k$ dependence of the self-energy using the perturbation theory up to the second-order terms. For the half-filling case they found that the first-order term made a large reduction of the $k$-mass.

On the other hand Ohkawa2d) suggested that in the vicinity of the Mott transition the formation of light quasi-particle could occur due to intersite quantum spin fluctuations using $1 / \mathrm{d}$ expansion method within the onsite Coulomb interaction.

The relation between these theories and real systems is not clarified enough now.

\subsection{Density of States}

From eq. (3.8)

$$
G_{\boldsymbol{k}}(\omega) \simeq \frac{Z_{\boldsymbol{k}_{F}}}{\left(\omega-E_{\boldsymbol{k}}\right)-\operatorname{Im} \Sigma_{\boldsymbol{k}}(\omega)} .
$$

If we assume the system can be described by the Fermi liquid theory, the coherent part of the spectral function is

$$
A_{\boldsymbol{k}}(\omega) \simeq Z_{\boldsymbol{k}_{F}} \delta\left(\omega-E_{\boldsymbol{k}}\right)
$$




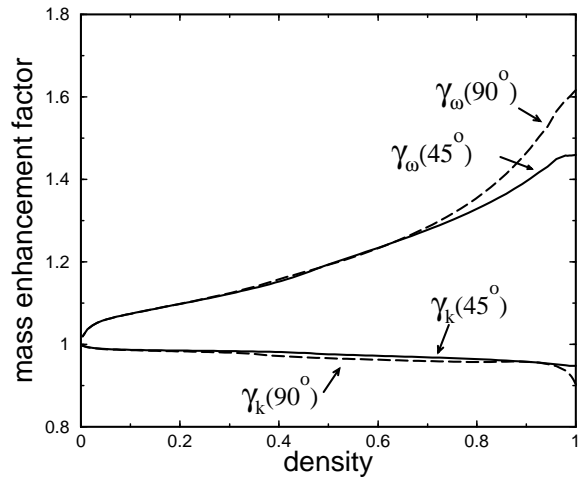

Fig. 8. $k$-mass and $\omega$-mass enhancement factors v.s. density of particles. $\gamma_{\omega}\left(90^{\circ}\right)$ denotes the $\omega$-mass enhancement factor at the Fermi surface in $(0, \pi)$ direction, and $\gamma_{\omega}\left(45^{\circ}\right)$ denotes the one in $(\pi, \pi)$ direction. $\gamma_{k}\left(90^{\circ}\right)$ and $\gamma_{k}\left(45^{\circ}\right)$ are the $k$-mass enhancement factors defined in the same way. Interaction strength $U=4$.

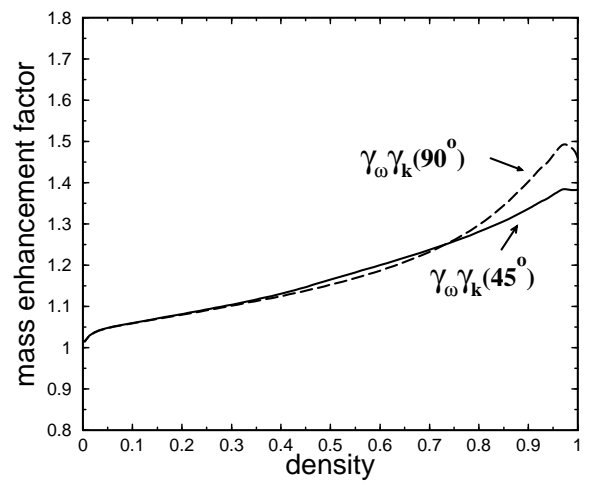

Fig. 9. Total mass enhancement factor v.s. density of particles. $\gamma_{\omega} \gamma_{k}\left(90^{\circ}\right)$ denotes the total mass enhancement factor at the Fermi surface in $(0, \pi)$ direction, and $\gamma_{\omega} \gamma_{k}\left(45^{\circ}\right)$ denotes that in $(\pi, \pi)$ direction. Interaction strength $U=4$.

Then the jump in the momentum distribution function across the Fermi surface is given by $Z_{\boldsymbol{k}_{F}}$. The density of states of the interacting system is defined as

$$
D(\omega) \equiv \frac{1}{N_{L}} \sum_{\boldsymbol{k}_{\sigma}} A_{\boldsymbol{k}}(\omega) .
$$

Putting eqs. (3.13) and (3.15) into eq. (3.16), we find that density of states at the Fermi level is related with the $k$-mass enhancement factor

$$
D(0) \simeq 2 \frac{1}{(2 \pi)^{2}} \int_{F S} \mathrm{~d} s \frac{\gamma_{k}}{v_{\boldsymbol{k}_{F}}^{0}},
$$

where $v_{\boldsymbol{k}_{F}}^{0} \equiv\left|\nabla \varepsilon_{\boldsymbol{k}_{F}}\right|$ and $\int_{F S} d s$ denotes the linear integration along the Fermi surface. If the system is isotropic in momentum space

$$
D(0)=\gamma_{k} D_{U=0}(0) .
$$

As shown in Fig. 10 the peak width of DOS shrinks as interaction increases, and the peak position of the DOS moves toward the Fermi level. These features resemble those in the infinite dimensional case 5 ) On the other hand DOS at the Fermi level is suppressed with the decrease in the $k$-mass enhancement factor. This feature is not found in the infinite dimensional case because the self-energy does not have $k$-dependence, and consequently DOS at the Fermi level remains the same as that of the non-interacting case. D)

Figure 11 shows the comparison of the DOS of SCSOPT with that of SOPT for $n=1$. In the case of SOPT the weight near the Fermi level decreases and two peaks build up at high energies. These two peaksare related with the upper and lower Hubbard bands.21, 12,12 On the other hand the SCSOPT DOS does not have such two peaks. The lack of high-energy resonance peaks in SCSOPT indicates that the SCSOPT is a weak coupling theory and is not correct for large $U$.

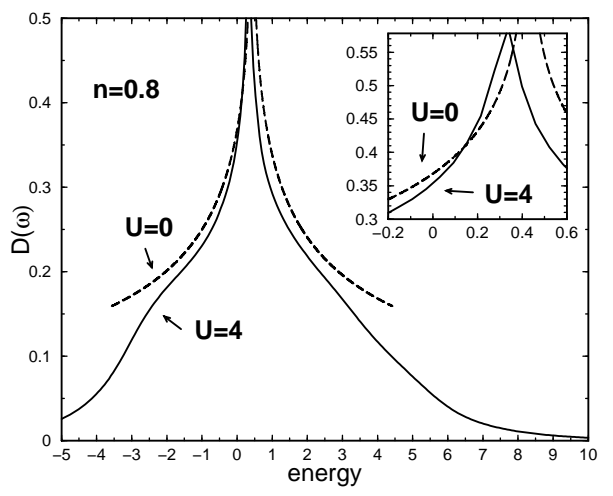

Fig. 10. Renormalized density of states versus energy for $n=0.8$ of the SCSOPT for interaction strengths $U=0$ and 4 . Inset shows the magnification near the Fermi level.

\subsection{Charge susceptibility}

If the chemical potential is represented by $\mu_{t}$, charge susceptibility $\chi_{c}$ is defined as $\mathrm{d} n / \mathrm{d} \mu_{t}$. Since we define $\mu$ as $\mu_{t}-U n / 2$ in $\S 2$, charge susceptibility is described as

$$
\chi_{c}=\frac{\mathrm{d} n}{\mathrm{~d} \mu} \frac{1}{1+\frac{\mathrm{d} n}{\mathrm{~d} \mu} \frac{U}{2}} .
$$

Charge susceptibility for $U=0$ is described as

$$
\chi_{c}^{0} \equiv \frac{\mathrm{d} n}{\mathrm{~d} \mu_{0}}=2 \frac{1}{(2 \pi)^{2}} \int_{F S} \mathrm{~d} s \frac{1}{v_{\boldsymbol{k}_{F}}^{0}} .
$$

For $U=0, \chi_{c}$ diverges logarithmically toward half-filling due to the Van-Hove singularity. 


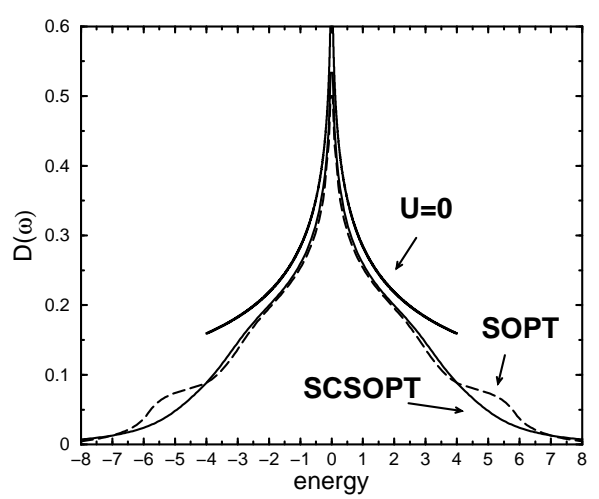

Fig. 11. Renormalized density of states versus energy for $n=1$ of the SCSOPT and the SOPT (second order perturbation theory) for interaction strength $U=4 . U=0$ case is also shown for comparison.

In the interacting case $\mathrm{d} n / \mathrm{d} \mu$ in eq. (3.19) is described in the Fermi liquid theory as 22

$$
\frac{\mathrm{d} n}{\mathrm{~d} \mu}=\sum_{\boldsymbol{k}_{\sigma}} \delta\left(E_{\boldsymbol{k}}\right) Z_{\boldsymbol{k}}\left[1-\frac{\partial \operatorname{Re} \Sigma_{\boldsymbol{k}}^{(s c)}(0)}{\partial \mu}\right] .
$$

Putting eq. (3.13) into eq. (3.21) we obtain

$$
\frac{\mathrm{d} n}{\mathrm{~d} \mu}=2 \frac{1}{(2 \pi)^{2}} \int_{F S} \mathrm{~d} s \frac{1}{v_{\boldsymbol{k}_{F}}^{0}} \gamma_{k} \gamma_{\mu},
$$

where we define

$$
\gamma_{\mu} \equiv 1-\frac{\partial \operatorname{Re} \Sigma_{\boldsymbol{k}}(0)}{\partial \mu} .
$$

As shown in Fig. 12 the charge susceptibility $\chi_{c}$ is suppressed by interaction near half-filling. This suppression is caused by the factor $\gamma_{\mu}$ in eq. (3.22), and can be thought as the precursor of the Mott insulator charge gap. Similar features have been reported in other theories which are in the framework of the Fermi liquid theory, such as the theory of infinite dimensional Hubbard model $\mathrm{B}$ and second order perturbatip theory for $\chi_{c}$ of the two dimensional Hubbard model.22

On the other hand our result is inconsistent with Furukawa and Imada's Quantum Monte Carlo study where $\chi_{c}$ diverges as $n$ goes to 1 .

\section{$\S 4$. Conclusion}

We have applied the self-consistent second order perturbation theory to study the two dimensional Hubbard model, which is a weak coupling theory and is expected to describe metallic phase of the model correctly. The SCSOPT satisfies the Luttinger sum rule exactly, which is necessary to construct the Fermi liquid theory.

First we have calculated the Fermi surface deformation, and the result shows that the interaction enhances the anisotropy of the Fermi surface near half-filling and

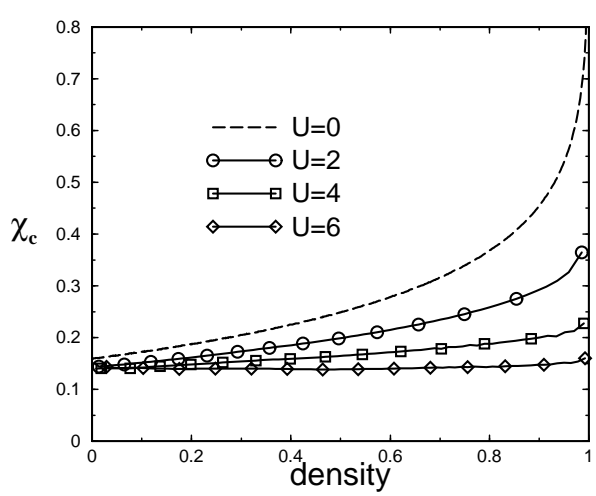

Fig. 12. Charge susceptibility $\chi_{c}$ as a function of the particle density.

reduces the anisotropy of it at lower particle densities.

The peak width of DOS shrinks as interaction increases, and the peak position of the DOS moves toward the Fermi level at non-half-filling densities. These features resemble those in the infinite dimensional case. On the other hand DOS at the Fermi level is suppressed with the decrease in $k$-mass enhancement factor. The decrease in $k$-mass enhancement factor is small and about 10 percent for $U=4$ at half-filling where it is maximum. This feature can not be found in the infinite dimensional case.

In the weak coupling theory the chemical potential shift induced by the interaction is related with the average of the $\omega=0$ self-energy over the non-interacting Fermi surface, and the Fermi surface deformation is related with the variation of it on that Fermi surface. These relations are well satisfied in SCSOPT for the two dimensional Hubbard model.

\section{Acknowledgements}

The author is indebted to Professor Y. Ōno for drawing attention to this problem and to Professor T. Matsuura and Professor Y. Kuroda for reading the manuscript and making a number of helpful suggestions. He also thanks T. Itakura and N. Nakamura for a lot of discussions.

[1] J. G. Bednorz and K. A. Müller: Z. Phys. B 64 (1986) 189.

[2] J. Voit: Rep. Prog. Phys. 57 (1995) 977.

[3] A. Georges, G. Kotliar, W. Krauth and M. J. Rozenberg: Rev. Mod. Phys. 68 (1996) 13.

[4] J. M. Luttinger: Phys. Rev. 119 (1960) 1153.

[5] E. Müller-Hartmann: Z. Phys. B - Condensed Matter 74 (1989) 507.

[6] J. E. Hirsch: Phys. Rev. B 31 (1985) 4403.

[7] N. Furukawa and M. Imada: J. Phys. Soc. Jpn. 60 (1991) 3604 .

[8] N. Furukawa and M. Imada: J. Phys. Soc. Jpn. 61 (1992) 3331.

[9] I. H. Inoue, I. Hase, Y. Aiura, A. Fujimori, Y. Haruyama, T. Maruyama, and Y. Nishihara: Phys. Rev. Lett. 74 (1995) 
2539.

[10] A. Ino, T. Mizokawa, K. Kobayashi, A. Fujimori, T. Sasagawa, T. Kimura, K. Kishio, K. Tamasaku, H. Eisaki and S. Uchida: Phys. Rev. Lett. 81 (1998) 2124.

[11] H. Schweitzer and G. Czycholl: Z. Phys. B - Condensed Matter 83 (1991) 93.

[12] V. Zlatić, K. D. Shotte and G. Schliecker: Phys. Rev. B 52 (1995) 3639.

[13] V. Zlatić, S. Grabowski and P. Entel: Phys. Rev. B 56 (1998) 14875.

[14] N. E. Bickers, D. J. Scalapino and S. R. White: Phys. Rev. B 62 (1989) 961.

[15] M. Langer, J. Schmalian, S. Grabowski and K. H. Bennemann: Phys. Rev. Lett. 75 (1995) 4508.

[16] A. A. Abrikosov, L. P. Gor'kov, and I. E. Dzyaloshinski: Methods of Quantum Field Theory in Statistical Physics, (Prentice-Hall, Englewood Cliffs, New Jersey, 1963).

[17] C. J. Halboth and W. Metzner: Z. Phys. B 102 (1997) 501.

[18] A. Sekiyama, T. Susaki, A. Fujimori, T. Sasaki, N. Toyota, T. Kondo, G. Saito, M. Tsunekawa, T. Iwasaki, T. Muro, T. Matsushita, S. Suga, H. Ishii and T. Miyahara: Phys. Rev. B 56 (1997) 9082.

[19] S. Yoda and K. Yamada: J. Phys. Soc. Jpn. 67 (1998) 1682.

[20] F. J. Ohkawa: Phys. Rev. B 57 (1998) 412.

[21] J. Hubbard: Proc. R. Soc. London A 281 (1964) 401.

[22] T. Hotta and S. Fujimoto: Phys. Rev. B 54 (1996) 5381. 